\title{
Multiple Time Scale Analysis of Precipitation in the Hengyang Basin
}

\author{
Qin JIANG ${ }^{1,}$ a, Yi-Min HUANG ${ }^{2, ~ b, *}$, Wei-Yue $\mathrm{LI}^{3, \mathrm{c}}$ and Jia-Hong WEN ${ }^{1, \mathrm{~d}}$ \\ ${ }^{1}$ Department of Geography, Shanghai Normal University, Shanghai 200234, China \\ 2 Department of City and Tourism Management, Hengyang Normal University, Hengyang, Hunan \\ 421002, China \\ ${ }^{3}$ Institute of Urban Study, Shanghai Normal University, Shanghai 200234, China \\ ajqshnu@163.com, ${ }^{b h y m i n 2004 @ s i n a . c o m ~}$ \\ clwy326@shnu.edu.cn,djhwen@shnu.edu.cn \\ ${ }^{*}$ Corresponding author
}

Keywords: Precipitation, Multiple time scale, Wavelet analysis, The Hengyang Basin

\begin{abstract}
This paper based on the monthly precipitation data of 9 surface meteorological stations in the Hengyang basin for 53 years (1960 to 2012), by using the methods of Morlet wavelet transform and Mexican hat wavelet analysis, the multi-time scales of precipitation of the Hengyang basin has analyzed. The results show that during the study period, the Hengyang basin has different time scales during the year and seasonal precipitation. The main periodic variation characteristics of quasi 24a, quasi 10a and quasi 3a time scales are existed in annual precipitation. And on the quasi 24a scale, the mutation points are 1963a, 1970a, 1977a, 1990a and 2004a. The characteristic period of spring and summer, or autumn and winter precipitation have highly similarity. And we speculate that the precipitation in the Hengyang basin tends to be less than normal in some time after 2012a.
\end{abstract}

\section{Introduction}

In recent years, wavelet analysis method has been widely used in climate change research [1]. Wavelet analysis has made significant achievements in studying the cyclical features of multi-time scales in climate change [2], correlation differences at different time scales [3] and mutability [4], it provides a new method for the objective research of multi-time scale structure and localized features of climate system change. In China, many scholars have used wavelet analysis to analyze/predict the time series of runoff, temperature and precipitation, discussed the precipitation characteristics of different regions in different time scales, Such as North China [5], the middle and lower reaches of the Yangtze River [6].

The Hengyang basin is the humid hilly land geomorphology unit of the subtropical zone in southern China. Due to the concentration of precipitation and heavy rain, the flood disaster is more serious. Previous studies have been conducted on the precipitation in the Hengyang basin, but most of them focus on analyzing the climate change characteristics and drought development trends [7], vulnerability assessment of water resources system [8], et al. In this paper, we have used wavelet analysis to reveal the characteristics of precipitation in the Hengyang basin, and provide scientific basis for the reasonable exploitation and utilization of rain-water resource and prediction of precipitation. 


\section{Study Region and Analysis Methods}

\section{Study Region}

The Hengyang basin is one of the six basins in Xiangjiang river valley, located in south-central Hunan province. The terrain is surrounded by high and medium low, and from southwest to northeast tilt (Fig 1). The area of basin area is about $15310 \mathrm{~km}^{2}$, the scope of roughly the same administrative region of Hengyang City, belonging to the subtropical monsoon climate zone. The inhomogeneous precipitation, both temporally and spatially. Average annual rainfall is about $1200-1400 \mathrm{~mm}$, the spring and summer seasons accounted for about $70 \%$ of the year, mountain precipitation is more than the ground [7].

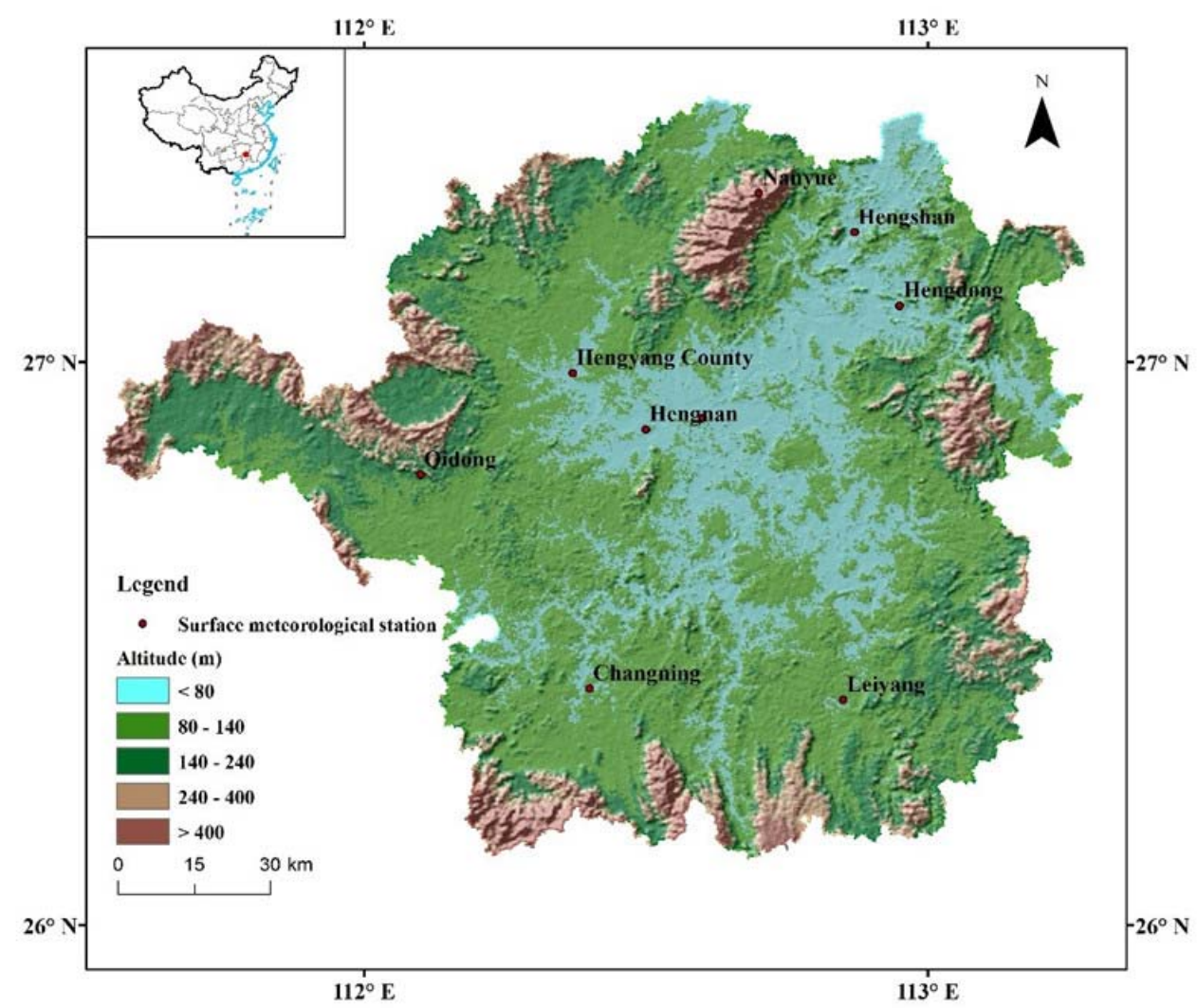

Fig. 1 the Hengyang Basin

\section{Data Sources}

The data used in this work includes the monthly precipitation data of 9 meteorological stations (Hengyang, Nanyue, Hengshan, Hengdong, Qidong, Hengyang, Changning, Leiyang and Hengnan) in Hengyang Basin for 53 years (1960 to 2012). Seasons divided as follows: spring (March to May), summer (June to August), autumn (September to November), and winter (December to the second year of February).

\section{Analysis Methods}

Wavelet analysis is the development and extension of Fourier analysis, with the characteristics of multi-resolution analysis, in the time and the frequency domain has the ability to characterize the 
signal localization. At the same time, it can reveal the multiple time scales of the hydrological series, describe the complexity of the sequence effectively, and analyze its internal detailed structure. In this paper, Morlet wavelet is used to analyze the characteristic time scale and periodicity of precipitation, and the Mexican hat wavelet is used to detect the possible mutation points at each scale. The multitime scale characteristics of annual precipitation and seasonal precipitation series are identified by wavelet transform coefficients, wavelet variance and wavelet transform coefficient sequences at different scales.

\section{Analysis of Result}

\section{Multi - Time Scale Characteristics of Precipitation}

\section{Analysis of Annual Precipitation}

The left panel of Figure 2 is the real part of the wavelet coefficients obtained from Morlet wavelet transform of the basin precipitation series from 1960 to 2012. The figure shows that the periodicity of the characteristic time scale of quasi-24a is obvious. The 1960-1966, 1980-1990 and 2006-2012 periods are negative phase (under the average years), 1967-1979, 1991-2005 are positive phase (above the average years), which indicates that the annual precipitation in the study period has undergone less-more-less-more-less than normal wet and dry alternation. On the quasi-10a scale, there are three obviously alternating cycles of rainfall abundance or deficiency in the 1960s to 1980s. However, in the 90's, the time scale no significant periodic oscillations. For the small scale below 5a, the abrupt point of precipitation increases and changes more frequently.

The right panel of Figure 2 is the wavelet variance reveals the main period of the annual precipitation series variation with time. Annual precipitation has three distinct peaks, which correspond to quasi-24a, quasi-10a and quasi-3a. The quasi-24a has the largest wavelet variance, which is the first main period of annual precipitation variation. Similarly, quasi-3a and quasi-10a are the second and third main periods. The fluctuation of the three main periods determines the variation characteristics of the annual precipitation over the whole time domain.

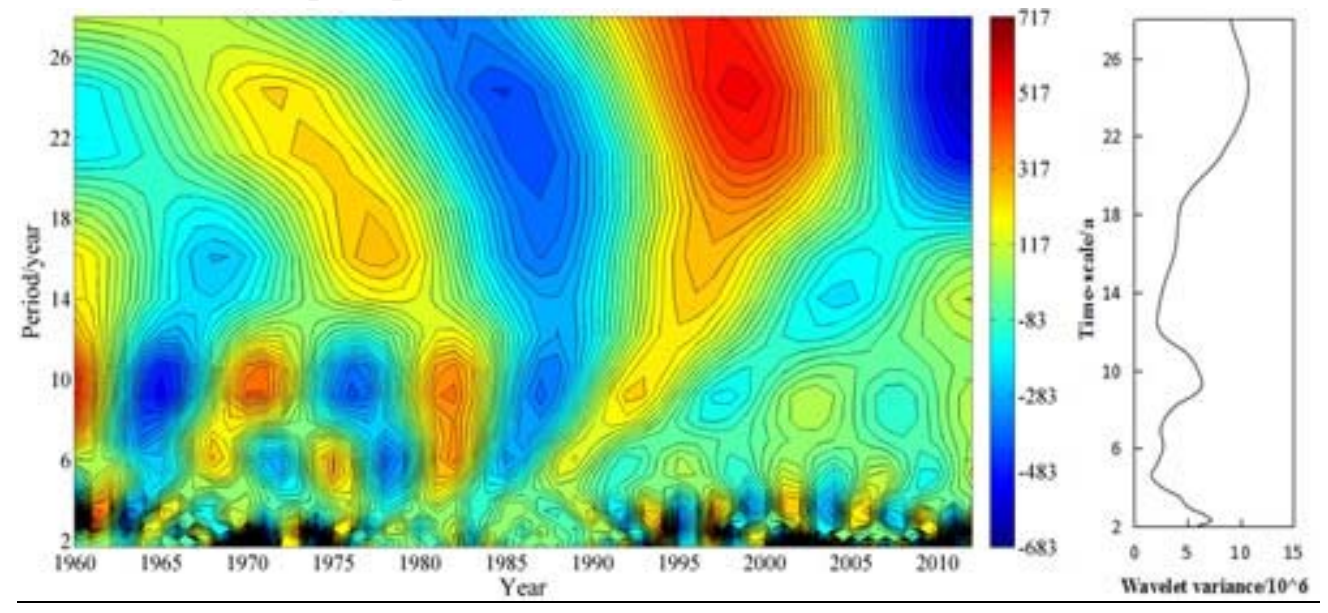

Fig. 2 the contours of the real part of Morlet wavelet coefficients (left panel) and Wavelet variance (right panel) of annual precipitation series

\section{Analysis of Seasonal Precipitation}

Figure 3 shows the contours of wavelet coefficients real part and wavelet variance in spring, summer, autumn and winter. The long-period oscillation of the precipitation sequence is obvious in the spring and summer seasons, and the scale center is quasi-24a. And generally experienced less-more-less- 
more-less than normal 2.5 cycles alternately. The oscillation signal of summer precipitation is especially strong after the 1970s, and the alternation period of dry and wet is about 10 years. But the signal is weaker in autumn and winter, and there is no closed contour, so the periodic variation of precipitation is not obvious, a longer sequence of data validation is required.

The right panel of Figure 3 illustrates the strength and distribution of precipitation over the four seasons at each time scale. Quasi-2a and quasi-24a scales of wavelet variance are the most significant in spring precipitation sequence, indicates that the main period of spring precipitation are quasi-2a, quasi-24a. In the same way, the main precipitation period in summer is $24 \mathrm{a}$, the main precipitation period are quasi-3a and quasi-5a in autumn, and the main precipitation period are quasi-2a and quasi$7 \mathrm{a}$ in winter.
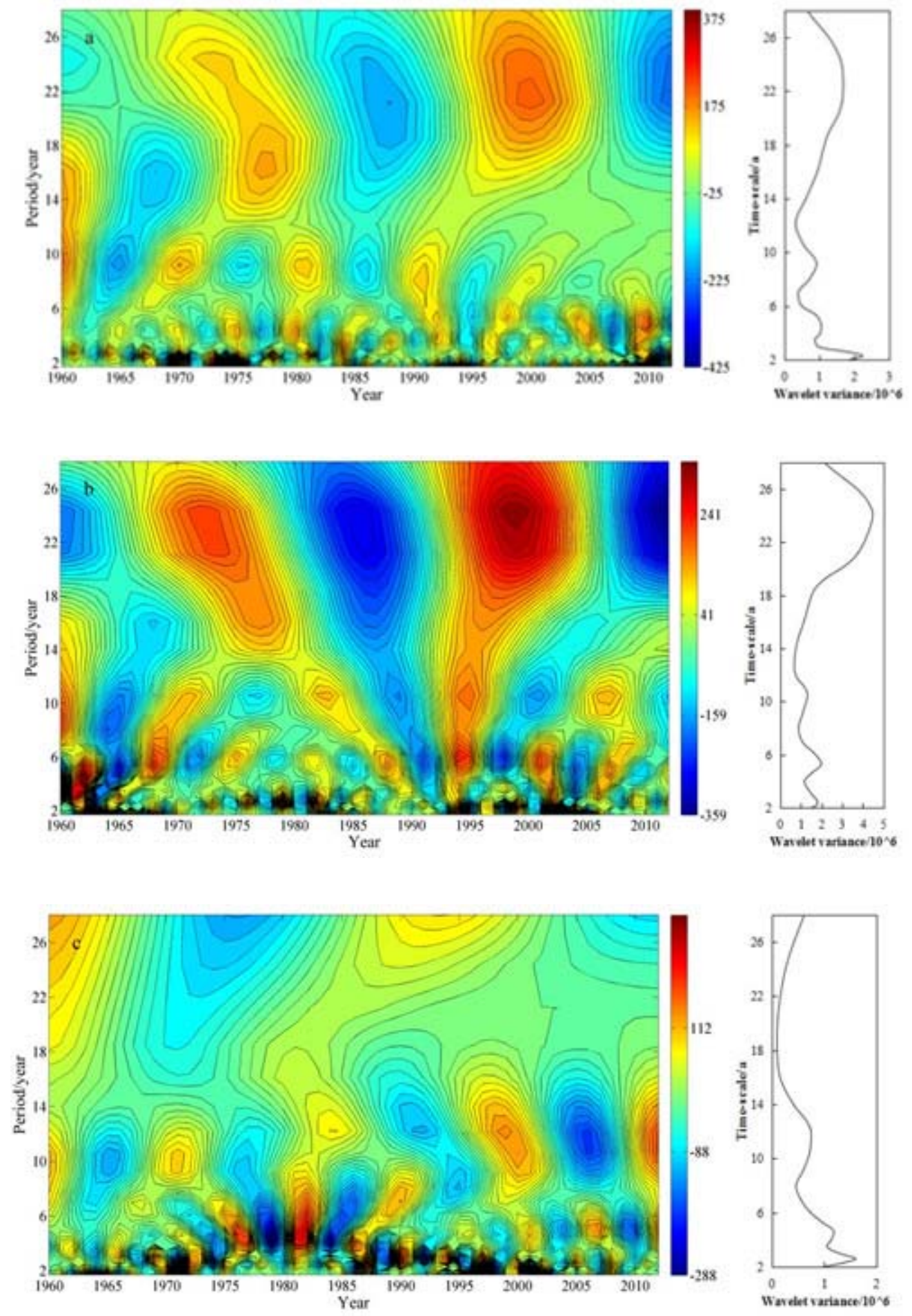

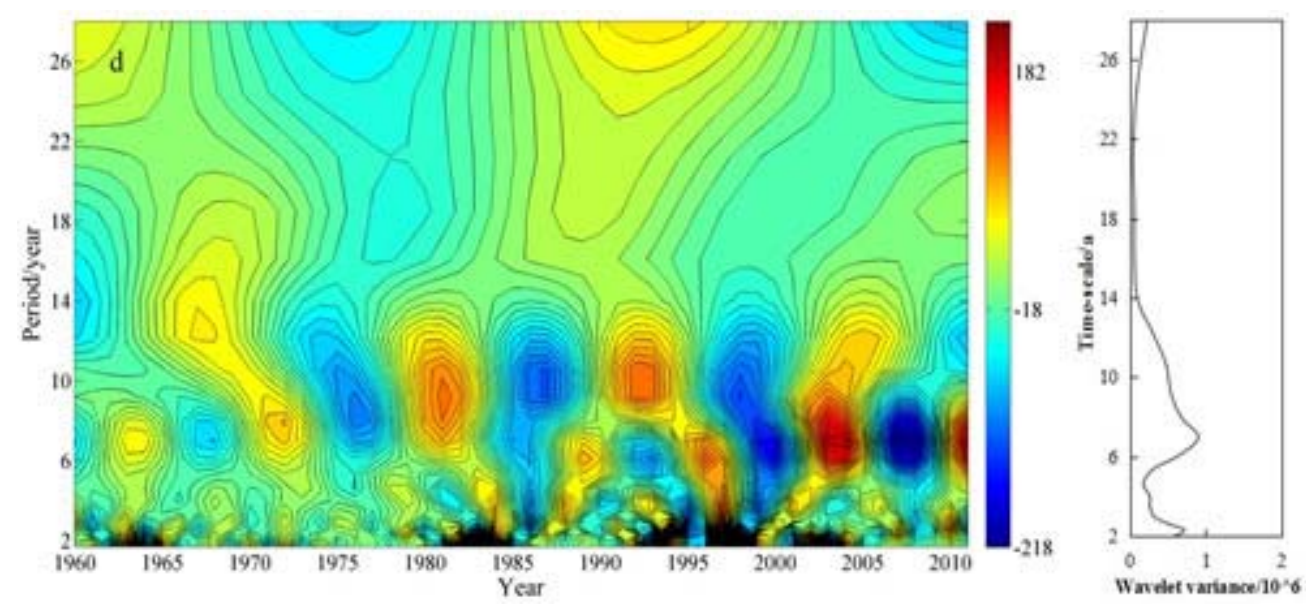

Fig. 3 the contours of the real part of Morlet wavelet coefficients (left panel) and Wavelet variance (right panel) of seasonal precipitation series

(a, b, c, d followed by spring, summer, autumn and winter, the same below)

\section{Mutation Point Analysis}

\section{Annual Precipitation}

Figure 4 shows the wavelet transform of the Mexican cap in the time series of the annual precipitation series. The positive and negative phases of wavelet coefficients correspond to more or less than average year precipitation, the zero value is the mutation point. On the quasi-3a scale, the annual precipitation is abundant and frequent alternately. In 53a, there are 14 dry-wet transition cycles, and the average conversion period is about 4a. On the quasi-6a and quasi-10a scale, the cycle of dry-wet transition is 8 times and 4 times respectively. On the quasi-24a scale, the mutation points are 1963, 1970, 1977, 1990 and 2004 year. It can be found from 1960 to 1963, 1970 to 1977, and 1991 to 2004 are the period of more than average year precipitation. From 1964 to 1969, 1978-1990, and 2005 to 2012 are the period of precipitation less than average. From this trend, we speculate that the precipitation in the Hengyang basin tends to be less than normal in some time after 2012a. Finally, it is concluded that the annual precipitation series is characterized by multiple time scales, the trend of dryness and wetness of precipitation is closely related to the scale size.

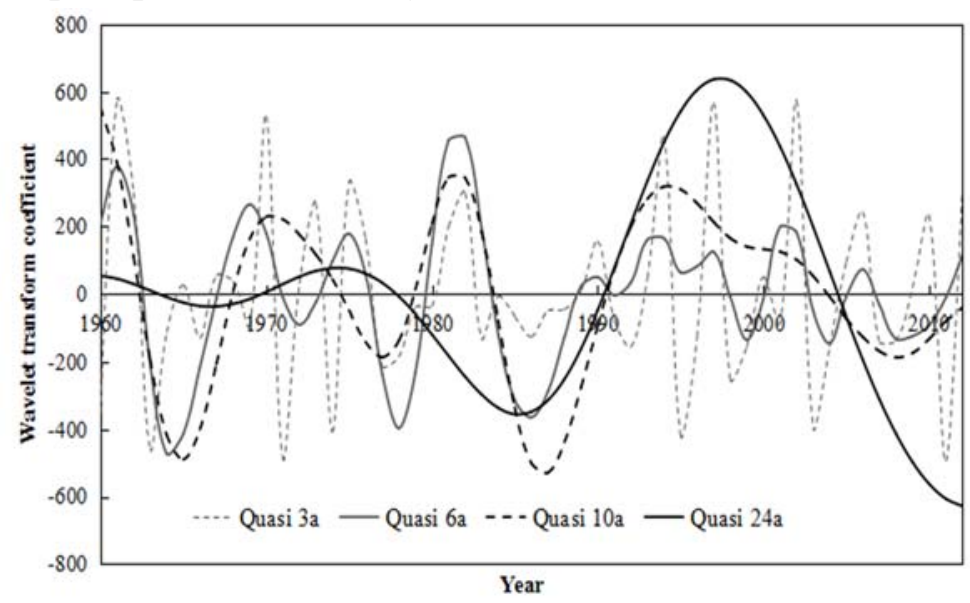

Fig. 4 the different time scales of Mexican wavelet transform coefficient curves of annual precipitation 


\section{Seasonal Precipitation}

Fig.5 are the Mexican wavelet coefficient transform curves of seasonal precipitation in different time scales. The mutation points of precipitation on the interdecadal scale are analyzed as follows:

The spring precipitation experienced two dry-wet transition cycles on the quasi-24a scale, mutation points are 1962, 1970, 1981, 1992 and 2005 year. Four mutation points in summer precipitation are 1967, 1978, 1991 and 2006 year on the quasi-24a scale. In autumn, 1969, 1973, 1985, 1991 and 2002 year are the rainfall precipitation reduction point (positive phase to negative phase), and 1969, 1980, 1989, 1996 and 2009 year, which precipitation increased abruptly (negative phase to positive phase), there are about 5 wet and dry transition periods in autumn precipitation, The frequency of winter precipitation is similar on quasi-7a scale and quasi-11a scale.
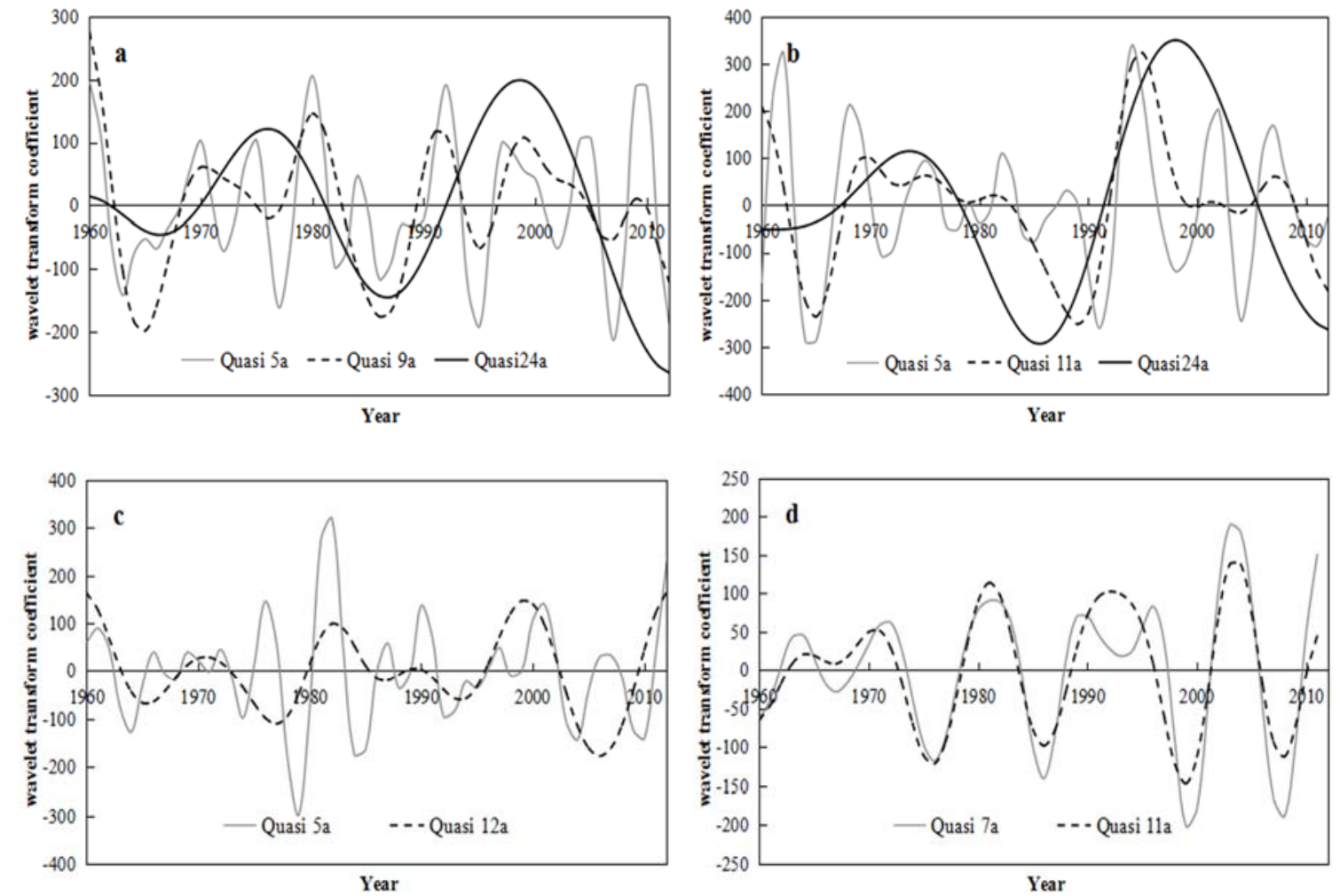

Fig. 5 the different time scales of Mexican wavelet transform coefficient curves of seasonal precipitation

\section{Summary and Conclusions}

Based on the continuous wavelet transform analysis of precipitation and seasonal precipitation in the last 53 years of the Hengyang basin, the main conclusions are as follows:

(1) In the last 53 years, the main periodic variation characteristics of quasi 24a, quasi 10a and quasi 3a time scales are existed in annual precipitation. On the quasi 24a scale, the annual precipitation has undergone less-more-less-more-less than normal wet and dry alternation. And we speculate that the precipitation in the Hengyang basin tends to be less than normal in some time after 2012a. The annual precipitation's wavelet variance is dominated by periodic variation on quasi-24a, quasi-10a and quasi3a scales. And on the quasi 24a scale, the mutation points are 1963a, 1970a, 1977a, 1990a and 2004a.

(2) The periodical oscillation of the spring and summer precipitation time series on the quasi-24a characteristic time scale is obvious, but the signal is weaker in autumn and winter, a longer sequence of data is required for verification. 
(3) In multi-time scales, there are different main periods and abrupt points of precipitation in different seasons. The overall performance of small-scale periodic changes nested in the large-scale periodic changes, the small-time variation of the scale is short, while the large-scale oscillation period is more obvious.

\section{References}

[1]Z. Z. HU, T. NITTA, Wavelet analysis of summer rainfall over North china and India and SOI using 1891-1992 data, Journal of the Meteorological Society of Japan. 74 (1996) 833-844.

[2]T.M. Feng, X. Zhang, and Y.P. Jing, Multi-Time Scale Analysis of Climate Variation in Qaidam Basin in Recent 46 years, Journal of Irrigation and Drainage. 33 (2014) 73-78.

[3]J.Y. Peng, Z.B. Sun, and G.R. Tan, Multi-Time Characteristics of Correlation between Chongqing Rainfall and Equatorial Eastern Pacific SST, Journal of Nanjing Institute of Meteorology. 22 (1999) 631-636.

[4]Y.F. Xue, Wavelet Analysis on Temperature and Precipitation in Zhanjiang and Shaoguan, Guangzhou Meteorology. 01 (2006) 28-32.

[5]L.P. Zhang, C.W. Zhu, and J. Xia, Analysis on the Change of Precipitation in Multiple Temporal Scales in North China, Arid Land Geography. 27 (2005) 549-552.

[6]Y.M. Zhang, X.G. Xun, and X.Y. Chen, Application of Wavelet Analysis to Climate Prediction on Drought and Flood Along the Middle to Lower Reaches of Yangtze River, Journal of PLA University of Science and Technology. 04 (2003) 90-93.

[7]L.F. Liu, Z.C. Xiao, and T. Chen, Climate change and drought trend in Hengyang basin over recent 33 years, Journal of Hunan Agricultural University (Natural Sciences). 40 (2014) 638-642.

[8]J. Zou, H.L. Liu, and W.W. Zhen, Study on Dynamic Characteristics of Artificial Vulnerability of Water Resource System in Hilly Areas of Southern China Based on GIS and RS-A Case Study on basin of Hengyang City, Resource Development \& Market. 31 (2015) 1327-1332. 